\title{
Influência da cinoterapia e perfil do animal durante exercícios fisioterapêuticos na Síndrome de Smith Lemli Optiza
}

Influence of cinoterapia and profile

of the animal during physiotherapy

exercises in Smith Lemli Opitz

FisiSenectus. Unochapecó Ano 3, n. 1 - Jan/Jun. 2015 Syndrome

Aline Dupont Menegazzo. alineee_menegazzo@hotmail.com Fisioterapeuta. Graduada pela Universidade Comunitária da Região de Chapecó Unochapecó.

Vanessa Stéfani Winck Souza. vanessast@unochapeco.edu.br Fisioterapeuta. Graduada pela Universidade Comunitária da Região de Chapecó Unochapecó.

Josilene S. Conceição. josilene.kaminski@unoesc.edu.br Fisioterapeuta. Mestre em Fisioterapia, Profa do Curso de Fisioterapia da Universidade do Oeste de Santa Catarina - Unoesc.

Franciane Barbieri Fiório. franciane.fiorio@unoesc.edu.br Fisioterapeuta. Doutora em Ciências da Reabilitação, Profa do Curso de Fisioterapia da Universidade do Oeste de Santa Catarina - Unoesc .

\section{Resumo}

A cinoterapia é uma modalidade de tratamento que utiliza o cão como facilitador para a realização das atividades, promovendo a saúde física por meio de mecanismos básicos que incluem a diminuição da solidão, depressão e ansiedade; aumento do estímulo para a prática de exercícios; melhora da saúde social, emocional e cognitiva; além dos efeitos no sistema nervoso simpático. No entanto, para a realização da atividade, o animal deve ter um perfil adequado. 0 presente estudo objetivou avaliar o efeito motivador da cinoterapia em uma criança com diagnóstico de Síndrome de Smith Lemli Optiz, bem como a influência do perfil do cão na execução dos exercícios. A pesquisa foi realizada com uma criança do sexo feminino, com quatro anos de idade. 0 protocolo de tratamento consistiu em alongamento dos músculos isquiotibiais e abdutores de quadril, estimulação à postura ajoelhada e ajoelhada com rotação de tronco, transferência do sentado para a ortostase, a ortostase e o deambular, nos quais foi analisado o tempo de realização medido por meio de filmagem. Para a avaliação da motivação foi realizado o teste adaptado de McAuley, Duncan \& Tammen (1987). Os resultados encontrados mostram que a cinoterapia teve influência positiva no tempo de realização dos exercícios e que o perfil do animal exerce influência negativa quando não adequado à realização da cinoterapia.

\section{Palavras-chave}

Terapia assistida por animais, Fisioterapia, Síndrome de Smith-Lemli-Opitz.

\section{Fisigenectus}




\begin{abstract}
The cinoterapia is a treatment modality that uses the dog as a facilitator for the achievement of activities, promoting physical health through basic mechanisms which include reduction of Ioneliness, depression and anxiety, besides the effects on the sympathetic nervous system and increase of incentive to the exercise practice, in addition emotional, cognitive and social health. Nevertheless, to perform the activity, the animal must have an appropriate profile. The research aimed to evaluate the motivational effect of citoterapia in a child diagnosed with Smith Lemli Syndrome, and the influence of the dog profile's in the execution of the exercises as well. The survey was conducted with a female child, four years old. The treatment protocol consisted of stretching the hamstrings muscles and hip abductors, kneeling posture and kneeling with trunk rotation stimulation, transferring from sat to orthostasis, the orthostasis and roam, where the realization time was analyzed through filming. For the motivation evaluation, McAuley, Duncan \& Tammen adapted test (1987) was used the. The results showed that cinoterapia had positive influence about realization exercise times, and the animal profile's had negative influence, when its profile is not appropriate for the citoterapia realization.
\end{abstract}

\title{
Keywords
}

Animal-Assisted Therapy, Physiotherapy, Smith-Lemli-Opitz Syndrome.

\section{Introdução}

$\infty \times \infty \times \infty \times \infty \times \infty \times \infty \times \infty \times \infty \times \infty \times \infty \times \infty \times \infty)$

A síndrome de Smith Lemli Optiz (SLOS) foi descrita, primeiramente, em 1964 por Smith et al., porém, sua etiologia só foi esclarecida em 1993, quando alguns autores descobriram o baixo nível de colesterol em pacientes afetados por essa síndrome ${ }^{1}$.

A SLOS é denominada como uma doença genética da biossíntese do colesterol, de transmissão autossômica recessiva e caracterizada por um padrão de dismorfias faciais (ptose, narinas antevertidas, micrognatia, fenda palatina, úvula bífida), alterações dos membros (sindactilia do segundo e terceiro dedos dos pés, polidactilia pós-axial) e malfomações congênitas múltiplas, incluindo pseudohermafrodismo no sexo masculino, hipolobulação pulmonar, agenesia renal, malformações cardíacas e cerebrais, bem como, atraso no crescimento, hipotonia neonatal e atraso mental ${ }^{1-3}$.

Nas crianças com disfunções neuromotoras, bem como na SLOS, devido ao atraso no desenvolvimento neuropsicomotor, há a necessidade de acompanhamento fisioterapêutico por longo período de tempo. Esse tempo prolongado de reabilitação, muitas vezes, faz com que as crianças apresentem resistência ao tratamento. Diante disso, faz-se necessária a implementação de novas opções de tratamento com objetivo de melhorar a aceitação ao tratamento específico, proporcionando a motivação e o prazer necessários para realizar os exercícios propostos pelo profissional da área.

Nesse sentido, a Terapia Assistida por Animais (TAA), tem a proposta de desenvolver um atendimento diferenciado a essas crianças, com o foco para o desenvolvimento do processo terapêutico centrado na relação estabelecida entre a criança e o animal. Os primeiros estudos a respeito dos efeitos da interação homemanimal demonstraram que a TAA pode promover a saúde física por meio de mecanismos básicos que incluem a diminuição da solidão, depressão e ansiedade, além dos efeitos no sistema nervoso simpático e aumento de estímulo para prática de exercícios, bem como a melhora de parâmetros relacionados à saúde social, emocional e funções cognitivas das pessoas ${ }^{4}$.

Diversos autores estudaram os benefícios proporcionados pela TAA na saúde humana, como aumento da autoestima, estimulação da memória, diminuição da pressão arterial sanguínea, do colesterol, da ansiedade, além da liberação de alguns neurotransmissores 
como dopamina, que age em regiões do cérebro promovendo, entre outros efeitos, a sensação de prazer e a motivação, e de -endorfina, que possui efeito em áreas cerebrais responsáveis pela modulação da dor, do humor, da depressão e da ansiedade ${ }^{5-7}$.

Uma das modalidades de TAA, a cinoterapia, terapia assistida por cães, teve sua origem no século XVIII, na Inglaterra, quando foi descoberto que o convívio com cães trazia benefícios psicológicos, pedagógicos e sociais ao seu praticante ${ }^{8}$.

Em seus estudos, Pecelin ${ }^{9}$ verificou que o cão torna o paciente mais susceptível ao tratamento, e que a cinoterapia demonstrou ter grande influência na melhora da cognição e qualidade de vida dos pacientes. Além disso, a motivação exercida pelo cão para a realização da fisioterapia estimula o paciente para a prática de exercícios ${ }^{10}$.

$\mathrm{Na}$ TAA são utilizados todos os tipos de animais que possam entrar em contato com o homem sem oferecer-lhe perigo controlado, como: cão, cavalo, gato, coelho, tartaruga, chinchila, hamster, peixe, pássaro, entre outros, no entanto, estudos relatam que a terapia realizada com animais que podem ser tocados obtém resultados mais eficientes, sendo assim, 0 principal animal utilizado é o cão, pois apresenta uma natural afeição pelas pessoas e é facilmente adestrado e capaz de criar respostas positivas ao toque, tendo grande aceitação ${ }^{4,6}$.

Para a escolha do animal, alguns critérios devem ser considerados, entre eles: o animal deve demonstrar obediência básica, ser calmo e colaborativo com as atividades propostas, ser receptivo a estranhos, permitir o toque e reagir com segurança a situações inesperadas. Podem ser usados cães de diferentes raças e portes, no entanto, deve-se tomar cuidado com a idade dos cães, pois animais muito jovens tendem a ser mais inquietos e os muito velhos se cansam muito rápido, por isso não são indicados ${ }^{4,6}$.

Ainda pouco conhecida no Brasil, a cinoterapia vem se firmando como uma grande aliada de profissionais de diversas áreas, tendo como proposta desenvolver um atendimento diferenciado aos pacientes, com o objetivo de facilitar a integração social e práticas educativas, uma vez que a terapia será realizada em ambiente adequado com contato direto com estímulos essenciais ao desenvolvimento neuropsicomotor e com o animal, sendo este o facilitador da terapia ${ }^{11}$.

Diante das alterações em relação ao desenvolvimento neuropsicomotor apresentadas pelos pacientes com sequelas neurológicas e dos benefícios proporcionados pelo animal como adjuvante em um processo terapêutico, torna-se de suma importância a investigação dos efeitos da cinoterapia e a interferência do perfil do animal sobre a prática de exercícios fisioterapêuticos.

Com isso, o objetivo deste estudo consiste em avaliar o efeito motivador da cinoterapia no tratamento fisioterapêutico de uma criança com síndrome de Smith Lemli Optiz, bem como verificar se o perfil do animal interfere na execução dos exercícios.

\section{Materiais e métodos}

$\infty \times \infty \times \infty \times \infty \times \infty \times \infty \times \infty \times \infty \times \infty \times \infty \times \infty \times \infty \times \infty)$

O presente estudo foi aprovado pelo Comitê de Ética em Pesquisa da Universidade Comunitária da Região de Chapecó sob o registro n 026/2011. 0 responsável autorizou a participação da criança no estudo e assinou o termo de consentimento livre e esclarecido.

0 estudo foi realizado com uma criança do sexo feminino, com quatro anos de idade com diagnóstico clínico de Síndrome de Smith Lemli Optiz, residente na cidade de Chapecó - SC. Não ocorreram intercorrências durante 0 período pré e peri natal dessa criança, no entanto, logo após o nascimento, esta apresentou quadro de hipotermia, tendo que ficar hospitalizada por 20 dias. Com o passar dos anos, a mãe observou que a criança não tinha o mesmo desenvolvimento das demais crianças da mesma idade, vindo assim a procurar auxílio médico. A criança foi tratada por diversas patologias, até que aos três anos de idade recebeu o diagnóstico clínico de Síndrome de Smith Lemli Optiz.

Para a realização da pesquisa foram utilizados dois cães da raça Labrador, de diferentes perfis, de propriedade do $2^{\circ}$ Batalhão do Corpo de Bombeiros 
de Chapecó, os quais estavam acompanhados de seus adestradores durante todas as atividades de cinoterapia.

Cão 1: Fêmea, seis anos de idade, calma e extremamente obediente ao seu comandante.

Cão 2: Macho, sete anos de idade, hiperativo e não obediente totalmente aos comandos do seu comandante. É um cão utilizado também para resgate, por isso é mais agitado.

Para caracterizar a história pré, peri e pós natal, a saúde geral da criança e os comprometimentos associados, bem como a avaliação do tônus postural e dos padrões de postura e movimento para a classificação da distribuição topográfica dos comprometimentos, foi utilizada uma ficha de avaliação construída para tal finalidade. A história pré, peri e pós natal foi fornecida pela mãe.

Para avaliação da motivação da criança frente às atividades propostas, foi utilizado o questionário de Avaliação da Motivação adaptado de McAuley, Duncan \& Tammen (1987), que foi respondido pelo responsável pela criança.

Esse questionário contêm oito questões que avaliam o interesse e o esforço/importância despendido à atividade, a escolha e o valor que essa atividade representa quem a está realizando. Para a pontuação da escala, dá-se uma nota e 1 a 7 para cada item, sendo que 1 a afırmação é pouco verdadeira, 4 a afirmação é em um nível intermediário de verdade e 7 a afirmação é muito verdadeira. A pontuação máxima é de 56 pontos. Esse questionário foi respondido pela mãe da criança, após a realização das atividades.

Com a avaliação física da criança, identificouse o tipo de tônus postural e os padrões de postura e movimento. Com isso, traçou-se os objetivos para o seu tratamento, constando: alongamentos musculares (isquiotibiais e abdutores do quadril), fortalecimento de tronco e estabilidade de cintura pélvica, sendo trabalhada para isso a postura ajoelhada (associada e não associada à rotação de tronco), a postura em pé, as transferências de sentado para em pé e a deambulação. Cabe ressaltar que foram utilizados materiais lúdicos para auxiliar na realização de todos os exercícios.

Foi estabelecido um protocolo de tempo para cada exercício, sendo: alongamentos - 1 minuto para cada grupo muscular; postura ajoelhada 4 minutos; postura ajoelhada com rotação de tronco - 4 minutos; passagem de sentado para em pé - 5 minutos; postura em pé - 5 minutos e deambulação - 5 minutos. Esse protocolo foi repetido nas três modalidades de tratamento em solo, com o cão 1 e com o cão 2, uma vez em cada modalidade.

Todos os exercícios, em todas as modalidades foram filmados com uma câmera digital (marca Sony HX1 9.1Mpx, zoom de 20X), para posterior análise e verificação do tempo de execução de cada exercício.

Nos exercícios de alongamento, para tomada de tempo, foi considerado o tempo de alongamento contínuo, pois a criança interrompia o alongamento, e a cada interrupção era iniciada nova tomada de tempo. Nos exercícios onde a criança deveria manter a postura ajoelhada, esta foi estimulada a permanecer com o auxílio de brinquedos em solo e do cão na cinoterapia. Nos exercícios de rotação de tronco na postura ajoelhada a criança foi estimulada a realizar a rotação com brincadeiras do seu interesse. No exercício de passar de sentado para em pé, a criança foi estimulada a sentar e levantar de forma lenta, usando como apoio a bola suíça ou o cão, dependendo da atividade.

O exercício que pretendia manter a criança na postura ortostática foi realizado em solo com o auxilio de um banco, onde eram colocados brinquedos.. Nessa postura, a pesquisadora promovia a estabilização no quadril da criança para que ela pudesse manter suas mãos livres e realizar as brincadeiras com os brinquedos ou com o cão. No exercício de deambulação em solo a criança recebeu estabilização com apoio em suas mãos e, durante a cinoterapia, a criança segurava com uma mão a guia do cão e com a outra a mão da pesquisadora.

Cabe salientar que, antes da realização das atividades na cinoterapia, foi realizada a aproximação da criança com os cães, para formação de vínculo. Em todas as atividades de solo e cinoterapia foi analisado o tempo de execução dos alongamentos e de permanência nas posturas. 
Os dados coletados foram analisados com auxílio do programa estatístico BioEstat 5.0. Inicialmente, foi realizada análise descritiva dos dados da amostra (médias e desvios padrões). Em seguida, foi aplicado o teste $t$ entre os grupos (solo, cão 1 e cão 2) para comparação dos tempos de execução dos exercícios. Em todas as análises foi adotado o nível de significância estatística de $5 \%(p<0,05)$.

\section{Resultados}

$\infty<\infty<\infty<\infty<\infty<\infty<\infty<\infty<\infty<\infty<\infty<\infty<\infty<\infty<\infty<\infty<$

O tempo de duração dos exercícios realizados em solo e com os cães de diferentes perfis foram analisados utilizando as filmagens e estão retratados na Tabela $\mathbf{1}$.

Observa-se que a média do tempo que a criança permaneceu nas atividades de alongamentos e de postura foi menor quando estas foram realizados em solo (149,9 seg). A média de tempo de realização dos exercícios com o cão 1 ficou muito próxima da média estabelecida pelo protocolo (173,7 seg e 173,3 seg, respectivamente). Já a média de tempo de realização dos exercícios com o cão 2 ficou um pouco abaixo da média estabelecida pelo protocolo (151,8 seg e $173,3 \mathrm{seg}$, respectivamente). Apesar de haver diferença entre as médias de tempo de realização dos exercícios, nas três situações (solo, cão 1 e cão 2) essa diferença não foi estatisticamente significativa, com $\mathrm{p}>0,05$.

Apesar de se observar que não houve diferença significativa entre as médias de tempo, pode-se observar que em alguns exercícios a média de tempo de execução foi bem abaixo do tempo estabelecido pelo protocolo, como é o caso dos exercícios de alongamento em solo, onde a média de tempo foi de $21,8 \pm 9,6 \mathrm{seg}$, contra uma média de tempo de protocolo de 60 seg. Já as médias de tempo dos alongamentos com o cão 1 e com o cão 2 ficaram acima da média estabelecida pelo protocolo, com $71,3 \pm$ $20,8 \mathrm{seg}$ e 70,8 $\pm 20,8 \mathrm{seg}$, respectivamente.

$\mathrm{Na}$ postura ajoelhada, observa-se que 0 tempo médio de permanência na postura com o cão 2 obteve a menor média, quando comparada com a mesma atividade em solo e com o cão 1 . Isso ocorreu devido ao fato de que o cão 2 era mais inquieto e não colaborava na realização da atividade, saindo de sua posição.

Na postura ajoelhada com rotação de tronco, observa-se que a média de tempo de execução da atividade ficou muito próxima nas três situações avaliadas (solo, cão 1 e cão 2), ficando próximas ao tempo estabelecido pelo protocolo.

A atividade de sentar e levantar e a postura em pé foram realizadas por mais tempo com o cão 1 e com o cão 2 do que em solo. Isso pode ser explicado pelo fato de a criança sentir-se motivada para a realização das atividades com os cães, as quais incluíam enfeitar os cães com laço de pelo, dar banho nos cães, entre outras.

Já a deambulação foi realizada por mais tempo em solo do que com os cães. Isso pode ser explicado pelo fato de a criança sentir-se mais segurança quando deambulou em solo, pois ela dava as duas mãos para a pesquisadora, com isso tinha mais estabilidade, já com os cães, uma das mãos estava na guia que conduzia o cão.

A Tabela 2 apresenta os escores da avaliação da motivação. Observa-se que de um total de 56 pontos que a avaliação poderia assumir, nas atividades em solo foi onde a criança apresentouse menos motivada, com escore de 36 pontos, e a modalidade que a criança apresentou-se mais motivada foi como cão 1 , com escore de 49 pontos. Apesar de haver diferença entre os escores nas três modalidades, essa diferença só foi estatisticamente significativa entre as modalidades solo e cinoterapia com cão 1 , com $p=0,013$. Na comparação entre as demais modalidades não houve diferença estatisticamente significativa dos escores.

Esses resultados sugerem que o cão exerce um efeito motivador para a realização dos exercícios. Observa-se que a média de tempo de realização dos exercícios com o cão 2 foi um pouco menor que a média de tempo com o cão 1 , isso pode ser explicado pelo fato de o cão 1 ser mais calmo, permanecendo nas posturas que seu adestrador o colocava, permitindo, assim, a realização do exercício. Já o cão 2 tinha um perfil agitado, corria muito, latia, assustando a 
criança, muitas vezes, e, durante os exercícios, saía da postura que seu adestrador o colocava, interrompendo, assim, a realização do exercício.

\section{Discussão}

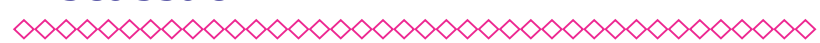

Enquanto modalidade terapêutica, a cinoterapia utiliza o cão como instrumento terapêutico, buscando desenvolver as potencialidades da criança, minimizando suas incapacidades com 0 intuito de proporcionar melhora da funcionalidade ${ }^{12}$.

Na cinoterapia, o cão torna-se especial pela capacidade que tem de transmitir alegria e cativar as pessoas, podendo, em um primeiro momento, ser um importante aliado na intervenção, principalmente no tocante à afetividade e à formação do vínculo e, posteriormente, com a participação mais ativa nos atendimentos ${ }^{4}$, o que foi observado na criança, que, no momento em que via o cão, antes mesmo da terapia, entrava em estado de euforia, abraçando e acariciando o cão.

Autores constataram em seus estudos que o convívio com o animal aumenta o interesse à prática de exercícios, relatam, ainda, que o ato de cuidar do animal favorece as atividades motoras, pois esse convivio exerce efeitos benéficos no comportamento afetivo, estabelecendo uma relação recíproca entre criança-cão ${ }^{13}$. No presente estudo, esse efeito motivador pôde ser percebido em todos os exercícios de um modo geral, visto que a criança permaneceu mais tempo realizando as atividades quando estimulada pelo cão do que quando somente com brinquedos em solo.

Com a interação dos cães, os exercícios, muitas vezes, nem eram percebidos pela criança, pois os cães a estimulavam as brincadeiras, as carícias, as conversas e o autocontrole, todos associados aos exercícios. Essa mudança de comportamento em relação à realização dos exercícios pode ser explicada por Buttram ${ }^{10}$ quando aborda a motivação exercida pelo cão para a realização da fisioterapia, sendo esta de grande estímulo para a realização de atividade física. Esse mesmo efeito motivador ou de melhora na adesão ao tratamento foi constatado por outros autores que realizaram a cinoterapia com crianças hospitalizadas. Nesses casos, as mães dessas crianças também relataram que estas esperavam ansiosas à chegada do cão, bem como relataram a melhora no quadro geral dessas crianças ${ }^{14-15}$.

A realização da fisioterapia assistida com animais em idosos institucionalizados também promoveu melhor interação com os idosos com o fisioterapeuta, melhorando a aceitação e diminuição da resistência ao tratamento específico, proporcionando a motivação e o prazer necessários para realizar os exercícios propostos pelo profissional da área ${ }^{16}$.

A sensação de prazer e motivação também foi descrita ${ }^{5}$ e tal sensação foi percebida no presente estudo quando a criança realizava o exercício da ortostase, no qual era incentivada pelo cão a permanecer nessa postura fosse para enfeitálo ou para jogar a bolinha de tênis para que este fosse buscar.

Além do efeito motivacional, autores relatam que o cão proporciona diversos outros benefícios, dentre eles a diminuição da solidão, da depressão, da ansiedade, da pressão arterial sanguínea, do colesterol, do controle da tensão e do estresse, bem como o aumento do estímulo para a prática de exercícios físicos, das células de defesa do organismo, melhorando, assim, o estado físico, educacional, motivacional, mental e afetivo $0^{4-7}$.

Não há raças específicas ou tamanho apropriado para o cão utilizado em terapia, porém, o que interfere é o comportamento deste, por isso deve haver uma seleção cuidadosa do animal a ser utilizado, envolvendo, por exemplo, testes de temperamento, que determinam como o cão vai reagir em novas situações, além dos testes de habilidades ${ }^{17}$.

0 animal deve estar adaptado ao paciente e às atividades, principalmente para que nenhum paciente sinta-se intimidado, com medo ou ameaçado pelo cão ${ }^{7}$. No presente estudo, as atividades com o cão 2 podem ter tido um menor desempenho devido a esses fatores, pois, apesar de o cão estar adaptado, pelo seu perfil hiperativo, algumas vezes, intimidava a criança, fazendo com que esta sentisse medo em algumas situações, não querendo realizar os exercícios. Nesse sentido, percebe-se que para que a terapia possa funcionar 
sem riscos, os animais participantes devem ser calmos, possuir o mínimo de adestramento e ter uma saúde rigorosamente controlada com vacinação e desvermifugação em dia ${ }^{18}$. A hiperatividade apresentada pelo cão 2 influenciou negativamente na realização dos exercícios, pois o animal distraía-se facilmente e saía do local da terapia.

Flôres ${ }^{19}$ comenta que todas as raças, de todos os tamanhos podem participar da atividade de cinoterapia, desde que tenham um temperamento adequado e realizem todos os testes e exames necessários. 0 autor recomenda, ainda, que a faixa etária do animal esteja entre um e nove anos, pois os filhotes podem ter dentes e unhas afiadas e necessidade de morder, além de serem mais frágeis, já os senis, normalmente, não são muito pacientes. Com isso, a faixa etária dos cães utilizadas no presente estudo foi levada em consideração, visando que não ocorressem os imprevistos citados acima.

Atualmente, o cão mais utilizado na terapia assistida por animais é o da raça Labrador, raça selecionada para a realização do presente estudo, pois esse tipo de cão apresenta-se como um grande aliado de crianças ou pessoas com alguma deficiência, servindo, por exemplo, como cão-guia e como aliado no trabalho de diversos profissionais. O contato com animais pode ser o ponto de partida para o desenvolvimento de diferentes habilidades tais como aprendizagem de conceitos, estimulação da linguagem, motricidade e expressão de emoções ${ }^{18}$.

Tendo em vista os benefícios obtidos com a interação entre as crianças e os cães, como abordado nos estudos mencionados e no presente estudo, acredita-se que a terapia assistida pelo cão é uma alternativa positiva de reabilitação física e mental em crianças com disfunção neuromotora, pois foi possivel observar que a ação de brincar associada a exercícios terapêuticos promoveu aumento da motivação e do prazer em realizar os exercícios fisioterapêuticos, melhor integração entre o fisioterapeuta e a criança e melhor aceitação aos exercícios.

A fisioterapia assistida por cães não substitui a fisioterapia convencional na realização de procedimento fisioterapêuticos que requer o contato direto do fisioterapeuta, como a realização de alongamento e mobilização passiva, normalização de tônus muscular/postural, dentre outros, no entanto, a presença do cão durante a fisioterapia convencional serve como um facilitador e estimulador das atividades, levando a um melhor resultado no tratamento.

Conclui-se com este estudo que a atividade com os cães mostrou-se eficaz para a realização dos exercícios quando comparada à realização dos exercícios em solo, pois promoveu um ambiente motivador para a criança, facilitando, assim, a realização dos exercícios.

Ainda, evidenciou-se que apesar de o cão exercer o efeito motivador para a realização dos exercícios, o perfil do cão pode levar ao maior ou menor sucesso na terapia, sendo que para um maior sucesso no tratamento, o ideal é que o cão seja calmo e colaborativo.

Como o aumento da demanda de pacientes com disfunções neuromotoras, torna-se cada vez mais importante e necessária a criação de novas formas terapêuticas para a melhora na qualidade do atendimento, aceitação e diminuição na resistência do tratamento e, diante dos resultados verificados nesta pesquisa, conclui-se que a cinoterapia permite às crianças com disfunções neuromotoras aprenderem novas tarefas e comportamentos, o que pode levar ao aumento do potencial para a resposta adaptativa necessária para a organização do cotidiano.

\section{Referências}

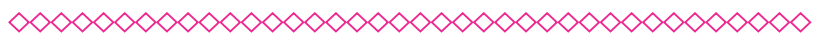

1. Silveira RN, Marchese DMA. A fisioterapia no tratamento motor e proprioceptivo na síndrome de Smith Lemli Optiz. In: Ciências da Saúde UNISA- Universidade de Santo Amaro. $12^{\circ}$ congresso de iniciação cientifica, $6^{\mathrm{a}}$ mostra de pós-graduação. São Paulo, 2009.

2. Vilar IG, Wanderley DC, Carvalho AT, Sant'anna ALH, Silva DP, Marques, MCA, et al. Síndrome de Smith-Lemli-Optiz: relato de caso In: XXII Congresso Brasileiro de GENÉTICA Médica, Salvador, 07 a 10 setembro 2010. Anais. 
3. Cardoso ML, Fortuna AM, Castedo S, Martins M, Montenegro N, Jakobs C et al . Diagnóstico prénatal de Síndrome de Smith-Lemli-Opitz. Arq Med. 2005 Jan;19(1-2): 23-27.

4. Dotti J. Terapis \& Animais. Atividade e Terapia Assistida por Animais para Organizações, Profissionais e Voluntários. 1 ed. São Paulo, Ed. Noética, 2005.

5. Araceli N. Pet Therapy: an effective approach to treatment. Alternative Medice. Period 11, 2003.

6. Kawakami CH, Nakano CK. Experiment report: animal assisted therapy (AAT) - another resource in the communcation between patient and nurse. In: Proceedings of the 8 . Brazilian Nursing Communication Symposium; 2002 May 02-03; São Paulo, SP, Brazil.

7. Mcguirk K. Animal- assisted therapy: at the children's specialized hospital. New Brunswick, 2001. April 26.

8. Oliveira GN. Cinoterapia: Benefícios da Interação entre crianças e cães. (2007). Disponível em: http://www.redepsi.com.br/portal/modules/ smartsection/item . php?itemid=524\&keywor ds=cinoterapia

9. Pecelin A, Furlan LA, Berbel AM, Lanuez FV. Influência da terapia assistida por animais em relação à cognição de idosos - estudo de atualização. Rev. ConScientiae Saúde. 2007;6(2):235-240.

10. Buttram D. Animal Assisted Therapy (AAT) as an Integral Part of Physiotherapy (PT) Sessions in a Nursing Home. In: $9^{\circ}$ International Conference On Humam-Animal Interactions, 2001, Rio de Janeiro. Abstract Book, Itália, 13 set., 2001, p. 36.

11. Amorim LJ, Silva LKJ, Rocha SD, Martin RT, Martin DH, Silva ARP, et al. Valorizando a vida e cidadania através da terapia facilitada por cães. In: Anais do III Seminário Internacional Sociedade Inclusiva PUC Minas. São José dos Campos, SP, 2004.

12. Severo JT. Equoterapia: A Equitação que promove saúde. In: Equoterapia: princípios e fundamentos básicos aplicados à saúde e à educação. 2a ed. Porto Alegre: [s.n.], 1998.
13. Fosco MM, Ribeiro PR, Ferraz FHA, Freitas Junior R; Martin DW, Raimundo CS et al. Aplicação da terapia assistida por animais no tratamento de crianças portadoras de paralisia cerebral - TAA Parte I. Saúde Coletiva. 2009; 32(6): 174-180.

14. Kobayashi CT, Ushiyama ST, Fakih FT, Robles RAM, Carneiro IA, Carmagnani MIS. Desenvolvimento e implantação de Terapia Assistida por Animais em hospital universitário. Rev. bras. enferm. 2009; 62(4): 632-636.

15. Ribeiro VF, Guede SCY. Fisioterapia assistida por animais em idosos Institucionalizados. In: Congresso brasileiro de atividade, educação e terapia assistida por animais, São Paulo, Anais, 14 e 15 jul. 2007, p. 32.

16. Fine AH. Handbook on animal-assisted therapy: theoretical foundations and guidelines for practice. 2006. (2nd ed., pp. 167-206). San Diego: Academic Press.

17. Alves AF; Comino, LS, Martinez RC, Prado LM, Manhoso FFR. Projeto Companheiro Animal: o médico veterinário e seu compromisso social através da zooterapia em crianças com necessidades especiais. Nosso Clinico. ANO 12 Nº6 - JAN/FEV 2009.

18. Flôres LN. Os benefícios da interação homem-animal e o papel do médico veterinário. Monografia apresentada a Universidade Federal Rural do Semi-Árido - UFERSA, Departamento de Ciências Animais para obtenção do título de especialista de Especialização em Clínica Médica de Pequenos Animais. Porto Alegre, 2009. 


\section{Anexos}

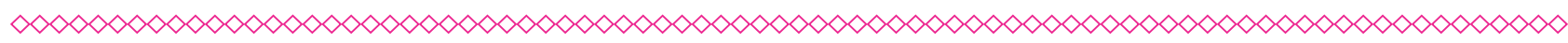

Tabela 1 - Tempo (segundos) de execução dos exercícios nas três situações de atividade e tempo estabelecido pelo protocolo de exercícios.

\begin{tabular}{|c|c|c|c|c|c|}
\hline Exercício & & \multicolumn{4}{|c|}{ Tempo em segundos (seg) } \\
\hline & & Solo & Cão 1 & Cão 2 & Protocolo \\
\hline \multicolumn{6}{|c|}{ Alongamento de isquiotibiais } \\
\hline & MID & 23 & 57 & 55 & 60 \\
\hline & MIE & 11 & 71 & 47 & 60 \\
\hline \multicolumn{6}{|c|}{ Alongamento de abdutores } \\
\hline & MID & 34 & 100 & 20 & 60 \\
\hline & MIE & 19 & 57 & 50 & 60 \\
\hline Ajoelhado & & 334 & 257 & 182 & 240 \\
\hline \multicolumn{2}{|c|}{ Ajoelhado com rotação de tronco } & 228 & 241 & 256 & 240 \\
\hline Sentar e levantar & & 238 & 278 & 310 & 300 \\
\hline \multicolumn{2}{|l|}{ Ortostase } & 206 & 321 & 239 & 300 \\
\hline \multicolumn{2}{|l|}{ Deambulação } & 256 & 181 & 207 & 240 \\
\hline \multicolumn{2}{|l|}{ Total $(\mu \pm D P)$ seg } & $\begin{array}{c}149,9 \pm \\
126,6\end{array}$ & $\begin{array}{c}173,7 \pm \\
104,5\end{array}$ & $\begin{array}{c}151,8 \pm \\
109,3\end{array}$ & $\begin{array}{c}173,3 \pm \\
110\end{array}$ \\
\hline
\end{tabular}

Fonte: dados das autoras (2011).

$M I D=$ membro inferior direito; $M I E=$ membro inferior esquerdo; $\mu=$ média; $D P=$ desvio padrão.

(clique para voltar ao texto)

Tabela 2 - Escore (número absoluto e porcentagem) da avaliação da motivação nas três diferentes situações de atividades.

\begin{tabular}{|l|l|}
\hline \multicolumn{1}{|c|}{ Modalidade } & \multicolumn{1}{c|}{ Escore $\left(n^{\circ}\right.$ absoluto e porcentagem) } \\
\hline Solo & $36(64,3 \%)$ \\
\hline Cinoterapia com cão 1 & $49(87,5 \%)$ \\
\hline Cinoterapia com cão 2 & $44(78,6 \%)$ \\
\hline
\end{tabular}

Fonte: dados das autoras (2011). 